\title{
Denken Sie auch an eine eosinophile Ösophagitis
}

— „Bei der eosinophilen Ösophagitis handelt es sich um eine chronische Antigen- bzw. immuninduzierte Öso-

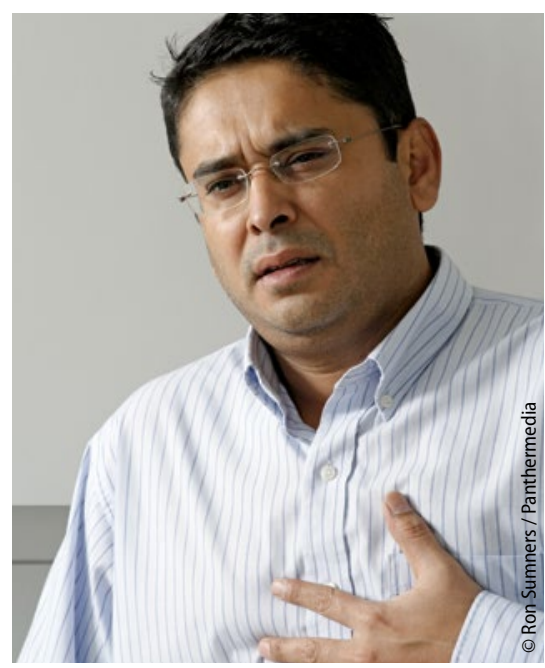

Hinter Thoraxschmerzen kann sich eine Ösophagitis verbergen. phaguserkrankung, die mit klinischen Symptomen verbunden und histologisch durch eine eosinophile Inflammation charakterisiert ist“, erläuterte Prof. Alex Straumann, Olten/Schweiz. Leitsymptom bei Erwachsenen ist die Dysphagie, aber auch eine Bolusimpaktion und die Angabe von Thoraxschmerzen sollten an diese Erkrankung denken lassen. Bei Kindern stehen unzureichendes Trinkverhalten und Regurgitationen im Vordergrund. „Der chronische Entzündungsprozess führt zu einer Fibrosierung und somit zu einer Versteifung der Ösophaguswand mit Motilitätsstörungen, Strikturen bzw. Stenosen“, so Straumann.

\section{Topische Steroide sind die Therapie der Wahl}

Mit topischen Steroiden wie Budesonid (Budenofalk ${ }^{\circledast}$ ) kann die Symptomlast deutlich reduziert werden. Vor allem das Risiko einer Bolusimpaktion nimmt deutlich ab. Darüber hinaus wird auch die Fibrosierung der Ösophaguswand gestoppt. „Steroide sind die einzigen Substanzen, für die eine Evidenz in Studien belegt ist", so Straumann.

Im Hinblick auf die Nebenwirkungen sollte eine topische Steroidtherapie der systemischen vorgezogen werden. Mit einer neuen Budesonid-Formulierung, genauer gesagt einer orodispersiblen Schmelztablette, konnte bei der Interimsanalyse der BUL-1-Studie bei 57,6\% der Patienten eine klinisch-histologische Remission erreicht werden. Die histologische Remissionsrate lag bei über $90 \%$.

\section{Dr. Peter Stiefelhagen}

- Pressegespräch „Eosinophile Ösophagitis und CED 2017 Chronisch entzündliche Erkrankungen des GI-Traktes adäquat behandeln", IX. Gastro-Conference; Berlin, Oktober 2017 (Veranstalter: Falk)

\section{Evidenzbasierte Phytotherapie}

\section{Reizdarmsymptome gehen zurück}

_ Viele Patienten wünschen sich heutzutage die Möglichkeit, ihre Erkrankungen pflanzlich zu behandeln - Ärzte hingegen setzten auf evidenzbasierte Medizin, sagte Dr. Jordi Serra, Barcelona. STW 5 (Iberogast ${ }^{\oplus}$ ), eine Kombination aus neun Heilpflanzenextrakten zur Behandlung bei funktionellen MagenDarm-Erkrankungen, wird beiden Wünschen gerecht. Die bewährte Kombination enthält neben Auszügen aus dem namensgebenden Iberis amara (Bittere Schleifenblume) unter anderem auch Extrakte aus Kamillenblüten, Kümmelfrüchten und Süßholzwurzel.

\section{Multi-Target-Prinzip}

Alle Komponenten der pflanzlichen Kombination wirken synergetisch und bringen die gastrointestinalen Funktio- nen wieder in Einklang, erklärte Prof. Peter Malfertheiner, Magdeburg. STW 5 steuere gleich mehrere Ziele im Verdauungstrakt an: Es wirkt antientzündlich, spasmolytisch sowie motilitätsfördernd und vermindert die Magenübersäuerung. So deckt STW 5 die oft sehr vielfältigen Symptome bei funktionellen Magen-Darm-Beschwerden ab.

Zudem tritt die Wirkung rasch ein, betonte Malfertheiner. Bereits 15-30 Minuten nach der Einnahme bemerken Patienten eine Besserung ihrer Symptome. STW 5 sei, was die Verträglichkeit betrifft, vergleichbar mit Placebo.

Katharina Grzegorek

- Presseveranstaltung "Iberogast ${ }^{\mathrm{Tm}}$. Harvesting the benefits of evidence-based phytomedicine in the treatment of Functional Gastrointestinal Disorders", UEG-Week; Barcelona, Oktober 2017 (Veranstalter: Bayer Vital)

\section{Einmal täglich dreifach gegen die COPD}

GlaxoSmithKline und Innoviva gaben kürzlich bekannt, dass die Europäische Kommission dem Arzneimittel Trelegy Ellipta (Fluticasonfuroat/Umeclidinium/Vilanterol [FF/UMEC/VI]) die Marktzulassung erteilt hat. Diese gilt für die Anwendung als Erhaltungstherapie bei erwachsenen Patienten mit moderater bis schwerer chronisch obstruktiver Lungenerkrankung (COPD), die mit einer Kombination aus einem inhalativen Kortikosteroid und einem langwirksamen $\beta_{2}$-Agonisten (LABA) nicht ausreichend behandelt werden können. Es handelt sich um die erste Tripletherapie für COPD-Patienten in ein und demselben Trockenpulverinhalator zur einmal täglichen Anwendung, die in Europa zugelassen ist. Enthalten ist eine Kombination aus einem inhalativen Kortikosteroid, einem langwirksamen Muscarinantagonisten (LAMA) und LABA.

Red.

- Nach Informationen von GSK 